\title{
Characteristics of Ketosis-onset Diabetes in Tunisian Population
}

\section{Melika Chihaoui*, Faouzi Kanoun, Nadia Tabassi, Bochra Ftouhi, Meriem Yazidi, Faiza Lamine and Hedia Slimane}

Department of Diabetology-Endocrinology, University Hospital La Rabta, Tunis, Tunisia

\begin{abstract}
Different types of diabetes can be presented by a ketosis (DK) or ketoacidosis (DKA). The objectives of this study were to analyze the characteristics of patients with newly diagnosed diabetes revealed by DK or DKA and discuss the different types of diabetes particularly ketone prone type 2 diabetes.

Subjects and methods: Retrospective study including 288 patients with new ketosis onset diabetes; 178 men, mean age: 35.5 years. We analyzed clinical, biological, therapeutic and subsequent clinical course data. Diabetes was classified according to clinical, biological and follow-up data.

Results: It was a DKA in 39 cases (13.5\%). Patients were classified as type 1 in $62.2 \%$ of cases and ketosis type 2 diabetes in $34.7 \%$ of cases. As compared to type 1 diabetes, ketosis type 2 diabetes patients had a more advanced age, higher body mass index and waist circumference, and less frequent DKA. After the acute episode, $60 \%$ of ketosis type 2 diabetes was treated with insulin and half of them had associated metformin. After a mean follow-up of 2.4 years, $52 \%$ of ketosis type 2 diabetes patients were treated with oral hypoglycemic agents.
\end{abstract}

Conclusion: Ketosis type 2 diabetes is a relatively new entity accounting for about one third of all new cases of DK or DKA in our population.

Keywords: Diabetic ketoacidosis; Ketosis prone type 2 diabetes; Type 1diabetes

\section{Introduction}

Ketosis is a frequent and potentially life-threatening complication of diabetes. It may occur in known diabetic patients or it may be a circumstance of diabetes diagnosis. Ketosis onset diabetes is generally considered and treated as type 1 diabetes. However, type 2 diabetes may be revealed by ketosis or keto-acidosis [1-4]. Classification of diabetes at the time of ketosis is important for a better long term management. The aim of this study was to analyze clinical, biological and subsequent clinical course data of subjects who have had a ketosis (DK) or ketoacidosis onset diabetes (DKA) and to discuss the different types of diabetes and especially ketosis-prone type 2 diabetes.

\section{Research Design and Methods}

We performed a retrospective analysis of all 288 patients referred for ketosis or ketoacidosis new onset diabetes to the endocrinology and diabetology department at Tunisian university hospital La Rabta from 1rst January 2000 to 31th October 2005. They were 178 males (61.8\%) and 110 females $(38.2 \%)$ with a mean (SD) age of 35.5 (17.1) years.

\section{We analyzed patient's medical records}

- Clinical data - Age, gender, consanguinity, family history of diabetes, known personal hypertension, dyslipidemia, autoimmune disease, clinical history (symptoms of hyperglycemia, their duration, precipitating factor), physical examination (general condition, temperature, Kussmaul breathing, symptoms of dehydration, state of consciousness, blood pressure, weight, height, waist circumference (WC), glucosuria, acetonuria)

- Para clinical data - Blood glucose, sodium, potassium, urea, creatinine, $\mathrm{pH}$ or bicarbonates,complete blood count, erythrocyte sedimentation rate, cyto-bacteriological examination of urine, chest radiography.

- Diabetes vascular complications (history of cerebro vascular or cardiac attack, blood pressure, peripheral pulses, neurological examination, optic fundus, electrocardiogram, proteinuria or microalbuminurie)

- Anti diabetic treatment prescribed initially and at follow-up.

Classification of diabetes was based on initial clinical and biological data (family history of diabetes, abdominal obesity, severity of polyuria and polydipsia, weight loss before DKA, duration of symptoms, severity of DKA, presence of vascular complications) and subsequent clinical course to last follow-up (weight, need for exogenous insulin). Patients were divided into two major groups; type 1 diabetes and ketosis type 2 diabetes.

Ketoacidosis is defined by hyperglycemia $>2.5 \mathrm{~g} / \mathrm{l}$, presence of urinary ketone $\geq++$ using Keto-Diastix Bayer ${ }^{*}$ ketone and glucose reagent strips for urinalysis and arterial $\mathrm{pH}<7.30$ or venous $\mathrm{HCO}_{3^{-}}<$ $15 \mathrm{mmol} / \mathrm{L}$. Ketosis was defined by the presence of hyperglycemia and urinary ketone without acidosis.

Data management and analysis were made using Epi-Info 6 statistical package. We used CHI 2 test for discontinued variables and Student's test for continues variables. The difference was considered significant if $\mathrm{p}<0.05$

\section{Results}

The study concerned 288 subjects with a sex ratio of 1.61 and a

*Corresponding author: Melika Chihaoui, Department of Diabetology-Endocrinology, University Hospital La Rabta, Tunis Endocrinology Jabbari, Tunis, Tunisia, E-mail: mm.chihaoui@planet.tn

Received October 29, 2011; Accepted Feberuary 20, 2012; Published Feberuary 25, 2012

Citation: Chihaoui M, Kanoun F, Tabassi N, Ftouhi B, Yazidi M, et al. (2012) Characteristics of Ketosis-onset Diabetes in Tunisian Population. J Diabetes Metab 3:175. doi:10.4172/2155-6156.1000175

Copyright: @ 2012 Chihaoui M, et al. This is an open-access article distributed under the terms of the Creative Commons Attribution License, which permits unrestricted use, distribution, and reproduction in any medium, provided the original author and source are credited. 
Citation: Chihaoui M, Kanoun F, Tabassi N, Ftouhi B, Yazidi M, et al. (2012) Characteristics of Ketosis-onset Diabetes in Tunisian Population. J Diabetes Metab 3:175. doi:10.4172/2155-6156.1000175

mean (SD) age of 35.5 (17.1) years [11-89 years]. Female were older than males (mean (SD) age of 38.7(18.6) vs 33.5(15.7) years, $\mathrm{p}=0.03$ ). Family history of diabetes was reported in $57.3 \%$ of cases. Parental Consanguinity was present in $36 \%$ of cases. Hypertension was known in 28 subjects (9.7\%), dyslipidemia in $24(8.5 \%)$ and an auto-immune disease in 5 patients (celiac disease in 1 case, Grave's disease in 1 case, hypothyroidism in 2 cases and vitiligo in 1 case). Polyuria and polydipsia were reported by 278 patients $(96.9 \%)$ during a mean (SD) period of 49.8 (93.5) days [4 days-2 years]. Weight loss was reported in 234 patients (81.5 \%) by an average (SD) of 7.1 (7.7) Kg. Mean body mass index (BMI) (SD) was $23.6(5.8) \mathrm{Kg} / \mathrm{m}^{2}$. Females had a mean BMI significantly higher than men (mean BMI (SD) 25.1 (6.2) vs 22.6 (5.2) $\left.\mathrm{Kg} / \mathrm{m}^{2}, \mathrm{p}=0.0003\right)$. Obesity $(\mathrm{BMI} \geq 30 \mathrm{Kg} / \mathrm{m} 2)$ was noted in 22 females $(12.3 \%)$ and 12 males (10.9\%). Overweight $\left(\mathrm{BMI}=25-29.9 \mathrm{Kg} / \mathrm{m}^{2}\right)$ was noted in 28 females (15.7\%) and in 37 males (33.6\%). Abdominal obesity was noted in 57 females (32\%) (WC $\geq 80 \mathrm{~cm}$ ) and in 34 males $(30.9 \%)$ (WC $\geq 94 \mathrm{~cm})$. It was a DKA in 39 cases $(13.5 \%)$ and a DK in 249 cases $(86.5 \%)$. Precipitating factor was an infection in $41.3 \%$ of cases, an emotional stress in $4.5 \%$ of cases and was not determined in the other cases. According to clinical and biological data, diabetes was classified as type 1 diabetes in 179 cases (62.2\%), ketosis type 2 diabetes in 100 cases $(34.7 \%)$ and other types in 9 cases (Figure1).

The comparison of clinical data between patients classified as type 1 diabetes and those classified as ketosis type 2 diabetes showed that type 1 diabetes patients were younger, more frequently of male gender (Figure 2,3), DKA was more frequent, BMI and WC were lower. The frequency of family history of diabetes didn't differ between the two groups (Table 1). Microvascular complications were present in 30 (30\%) ketosis type 2 diabetes patients: retinopathy in 6 cases (6\%), peripheral neuropathy in 21 cases $(21 \%)$ and nephropathy in 7 cases (7\%). Hypertension was present in 28 cases (28\%), cerebro-vascular attack in 4 cases (4\%), coronary heart disease in 7 cases (7\%) and peripheral artery disease in 4 cases (4\%).

At discharge, insulin therapy was prescribed in all type 1 diabetes patients and in $60(60 \%)$ ketosis type 2 diabetes patients in whom 30 associations with metformin. The others were treated with oral anti-diabetic agents (Figure 4). All ketosis type 2 diabetes patients presenting with DKA were treated with insulin. Among the 73 ketosis type 2 diabetes patients followed at the office, during a mean followup period (SD) of 2.4 (1.1) years, 28 patients were under oral drugs and 10 over the 45 patients $(22.2 \%)$ initially treated with insulin were switched to oral drugs after a mean period of 14.7 months. Thus, $52 \%$

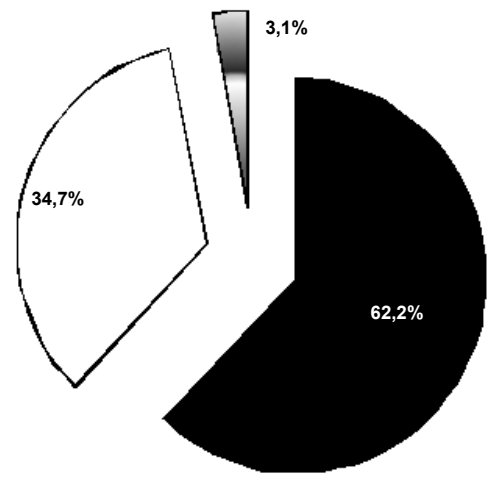

- type 1 diabetes aketosis type 2 diabetes -other types

Figure 1: Classification of Diabetes.

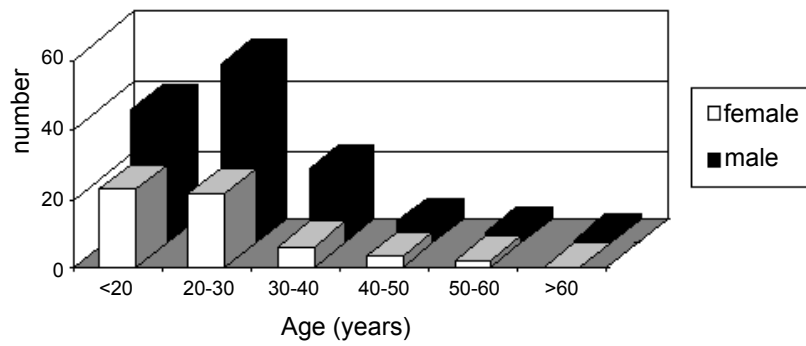

Figure 2: Distribution of patients according to age and gender in type 1 diabetes.

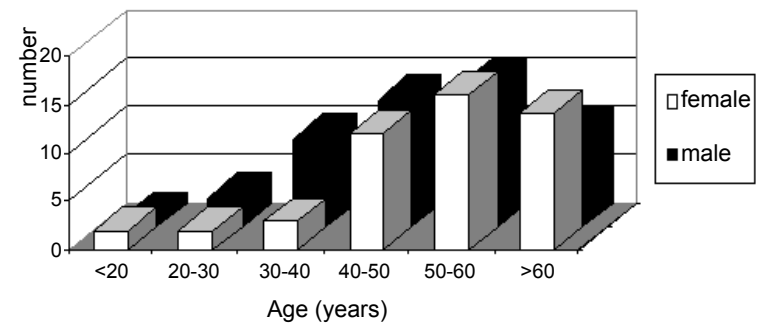

Figure 3: Distribution of patients according to age and gender in ketosis type 2 diabetes.

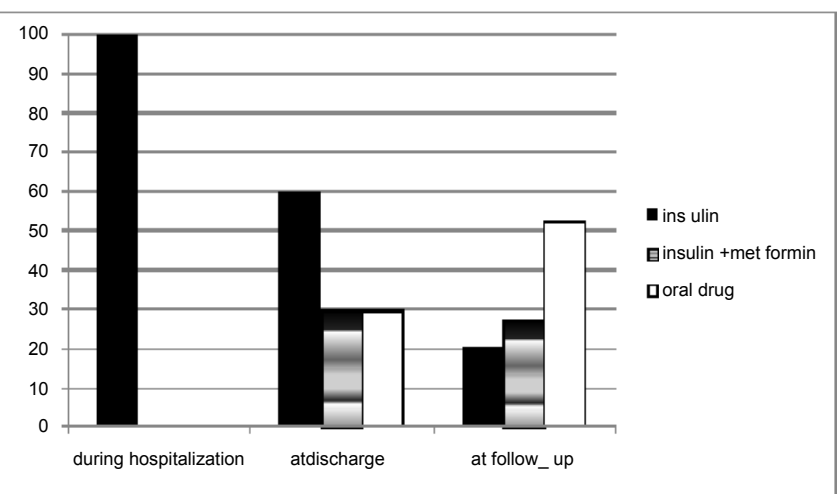

Figure 4: Treatment of ketosis type 2 diabetes.

of ketosis type 2 diabetes patients followed at the office were treated with oral drugs.

\section{Discussion}

Type 1 diabetes is characterized by a deficiency in insulin secretion and has generally an acute clinical presentation, whereas type 2 diabetes is characterized by an insulin resistance and is a latent disease that can be asymptomatic for years. However, type 2 diabetes can be expressed by a ketosis in the presence of a precipitating factor but ketosis is not a classic initial presentation of the disease. Type 2 diabetes generally occurs in patients with family history of diabetes, abdominal obesity and can be associated with hypertension and dyslipidemia and complicated by micro and macroangiopathy. Whereas, type 1 diabetes generally appears in younger subjects with normal weight and is presented with a marked polydipsia and polyuria, an important weight 


\begin{tabular}{|c|c|c|c|c|}
\hline & $\begin{array}{l}\text { All patients } \\
(\mathrm{n}=288)\end{array}$ & $\begin{array}{l}\text { Type } 1 \text { diabetes } \\
(\mathrm{n}=179)\end{array}$ & $\begin{array}{l}\text { Ketosis type } 2 \text { diabetes } \\
(n=100)\end{array}$ & $\begin{array}{l}\text { P (type } 1 \text { vs ketosis type } 2 \\
\text { diabetes) }\end{array}$ \\
\hline Age (years) & $35.5(17.1)$ & $25.9(10.0)$ & $51.4(14.6)$ & 0.000000 \\
\hline Gender ratio (male/female) & 1.61 & 2.2 & 1.04 & 0.005 \\
\hline Ketoacidosis & 13.5 & 17.3 & 8.0 & 0.02 \\
\hline Family history of diabetes & 57.3 & 56 & 65 & NS \\
\hline Known hypertension & 9.7 & 0 & 28 & 0.0000000 \\
\hline Infection & 41.3 & 36.8 & 49.0 & 0,04 \\
\hline Weight loss (Kg) & $7.1(7.7)$ & $6.9(6.7)$ & $7.6(9.5)$ & NS \\
\hline Weight variation & -12 & -13 & -11 & NS \\
\hline BMI $\left(\mathrm{Kg} / \mathrm{m}^{2}\right)$ & $23.6(5.8)$ & $20.8(4.1)$ & $28.5(5.1)$ & 0.000000 \\
\hline Obesity & 11.8 & 2.8 & 29 & 0.000000 \\
\hline Overweight & 22.2 & 8.9 & 46 & 0.000000 \\
\hline Waist circumference (cm) & $85.8(15.4)$ & $78.1(12.1)$ & $98.1(12.2)$ & 0.000000 \\
\hline Abdominal obesity (TT> $94 \mathrm{~cm}$ in men and $>80 \mathrm{~cm}$ in women) & 31.6 & 12.8 & 65 & 0.0000000 \\
\hline
\end{tabular}

Values are mean (standard deviation) or percentage

Table 1: Clinical characteristics of patients according to the type of diabetes

loss and a ketoacidosis. Type 2 diabetes can be treated with oral agents but type 1 diabetes necessitates insulin therapy. We used a series of these arguments for the classification of our diabetic patients. Thus, $37.8 \%$ of cases with presenting DK and DKA revealed ketosis prone type 2 diabetes. This particular form of diabetes was reported in some populations such as those of black African origin and some ethnic minorities. In African Americans, Umpierrez [1] noted that $74 \%$ of patients with inaugural DK or DKA had ketosis type 2 diabetes. On the same way, Nyenwe et al. [2] and Pinero-Pilona \& Raskin [3] noted a frequency of $60 \%$. In Koreans [4], this sub-type of diabetes represented $43 \%$ of inaugural DK or DKA. Ketosis type 2 diabetes represents one over 8 cases of diabetes in black American people from African origin [2,5-8] but is rarely described in Asiatic and white populations [9]. Our population is particular as it is a mixture of Arab and Berber races and mostly originates from rural areas that migrated to the capital city of Tunis.

Mean age of patients with ketosis type 2 diabetes was 51.4 years in our study and varied from 33 to 53 years in the literature $[5,10]$. All studies agree that type 1 diabetes appears at an earlier age. However, age is not sufficient to distinguish between the two types of diabetes as the two types can occur at any age.

Male gender was more frequently described in this sub type of diabetes (gender ratio varying from 1.5 to 3 ) but this was not the case in our study (gender ratio was 1.04) [8,11]. Family history of diabetes was as frequent in the two types of diabetes in our population $(56 \%$ and $65 \%$ ) and is explained by the high prevalence of diabetes in our general population [12]. So, the presence of type 2 diabetes in relatives doesn't exclude type 1 diabetes. Family history of diabetes in ketosis type 2 diabetes was also common in other populations varying from 60 to $84 \%[10,13]$.

Hypertension was present in $28 \%$ of our ketosis type 2 diabetes patients which is close to that noted in other studies like that of PineroPilona et al. [11]. DKA was less frequent (8\%) than DK in our ketosis type 2 diabetes. Tanaka et al. in Japan [14] reported $45.8 \%$ of DKA in ketosis type 2 diabetes and explained this high frequency of DKA by a pancreatic glucotoxicity secondary to an excess of absorption of soft drinks. On the other hand, ketosis type 2 diabetes represented $20.5 \%$ of all cases of DKA in our study. So, the severity of a DKA doesn't exclude ketosis type 2 diabetes.

Obesity is a classic feature of type 2 diabetes and is the main cause of insulin resistance. BMI in our ketosis type 2 diabetes was significantly higher than in type 1 diabetes (mean (DS) BMI of 28.5 (5.1) $\mathrm{Kg} / \mathrm{m}^{2}$ vs $\left.20.8(4.1) \mathrm{Kg} / \mathrm{m}^{2}, \mathrm{p}=0.0000\right)$ and varied from 26 to $37 \mathrm{Kg} / \mathrm{m}^{2}$ in different series [2,8]. $29 \%$ of our ketosis type 2 diabetes patients were obese and $46 \%$ were overweight. The frequency of obesity in ketosis type 2 diabetes varied from $20.6 \%$ in the study of Mauvais Jarvis et al. [13] to $55 \%$ in the study of Banerji et al. [15] and $81.5 \%$ in that of PineroPilona et al. [11].

Different precipitating factors can lead to hyperglycemic crisis. Infectious diseases are the most common cause occurring in 30 à 50 $\%$ of cases [16]. A precipitating infectious cause was found in $36.8 \%$ of our type 1 diabetes patients and $49 \%$ of ketosis type 2 diabetes. Balasubramanyam et al. [7] reported that half of ketosis type 2 diabetes presented by DKA had no identifiable precipitating factor. Glucotoxicity and lipotoxicity might be implicated in the insulinopenia causing DKA in ketosis type 2 diabetes $[2,14,17,18]$. Glucotoxicity can be secondary to an excess of soft drinks taken to satisfy thirst [19]. Sobngwi suggested the role of human herpes virus 8 (HHV-8) in the pathogenesis of ketosis type 2 diabetes [20].

Distinguishing type 1 from ketosis type 2 diabetes is generally easy. A young age, a normal weight, the absence of a precipitating factor, the severity of ketoacidosis and the presence of an autoimmune disease are in favour of type 1 diabetes. Whereas, an overweight, the presence of hypertension or of vascular complications and the possibility of treatment with oral drugs are in favour of ketosis type 2 diabetes $[5,10]$.

However, there are some situations where it is difficult to distinguish between the two types of diabetes with only these clinical data. So, the search for the presence of pancreatic auto antibodies and the evaluation of the secretary capacity of beta cells by the dosage of C-peptide can be helpful. Unfortunately, these biological parameters are not routinely available in our country. A Tunisian prospective study concerning 63 cases of ketosis onset diabetes occurring after 30 years of age revealed that pancreatic auto-immunity was present in $36.5 \%$ of cases and that Beta-cell functional reserve was preserved in $54 \%$. With these data diabetes can be classified by the $\mathrm{A} \beta$ scheme based on the presence or absence of $\beta$-cell autoantibodies (A+ or A-) and $\beta$-cell functional reserve $(\beta+$ or $\beta-)$ [21].

Ketosis type 2 diabetes is a sub type of diabetes that is intermediate between type 1 diabetes and type 2 diabetes. It is characterized by an acute initial presentation with severe hyperglycemia and ketosis indicating an acute insulinopenia as type 1 diabetes, but it concerns older and more corpulent subjects without auto-immune markers 
Citation: Chihaoui M, Kanoun F, Tabassi N, Ftouhi B, Yazidi M, et al. (2012) Characteristics of Ketosis-onset Diabetes in Tunisian Population. J Diabetes Metab 3:175. doi:10.4172/2155-6156.1000175

[15]. After the acute episode, it has the clinical course of classical type 2 diabetes and can be treated without insulin [10]. This sub type of diabetes had been named differently as 'flatbush diabetes' [15] or 'ketosis prone type 2 diabetes' [6,13].

After the acute episode, $60 \%$ of our ketosis type 2 diabetes patients were treated with insulin. It was the case in $61 \%$ in the study of PineroPilona et al. [3], 42.8\% in that of Banerji et al. [15] and47\% in that of Maldonado et al. [21], where as in only $31.8 \%$ of patients in the study of Hsin Yu et al. [22] and 24.3\% in the study of Mauvais-Jarvis et al. [13].

A re-evaluation of beta cell secretion's capacity might indicate the necessity of continuing insulin therapy. The management of our patients was only based on clinical data, and $52 \%$ of our ketosis type 2 diabetes followed at the office was treated with oral hypoglycaemic drugs. In the study of Pinero-Pilona et al. [11], $39 \%$ (21/54 cases) of DKA were treated with oral drugs after a mean follow-up of 12 months. Mauvais-Jarvis et al. [13] described a remission of diabetes in $75.7 \%$ of African sub-Saharan patients presented with DK or DKA, after a period of 14.3 months of insulin therapy. Forty percent of patients didn't necessitate insulin therapy during a follow-up of 10 years. It has been reported that treatment with oral hypoglycaemic drugs can extend the duration of the remission phase and prevent ketosis relapse $[13,23]$. This reversible dysfunction of beta cells is variable [24]. This remission is different from 'the honey moon' of type 1 diabetes that has a shorter duration. So, insulinopenia observed in the acute phase is transient in this subtype of diabetes. An initial insulin therapy can relieve glucotoxicity and restore beta cell function. The patients can later be treated with oral drugs like classic type 2 diabetes [25].

In conclusion, ketosis or ketocidosis generally reveals type 1 diabetes but can also reveal ketosis prone type 2 diabetes. Based on clinical data, $34.7 \%$ of our ketosis or keto-acidosis onset diabetes was ketosis prone type 2 diabetes. In 52\% of whom insulin therapy was subsequently withdrawn. This sub type of diabetes has been described in different populations especially in ethnic minority groups. It is characterized by a transient insulinopenia that is reversible after a short period of insulin therapy improving beta cell function.

\section{References}

1. Umpierrez GE (2006) Ketosis-Prone Type 2 Diabetes: Time to revise the classification of diabetes. Diabetes Care 29: 2755-2757.

2. Nyenwe E, Loganathan R, Blum S, Ezuteh D, Erani D, et al. (2007) Admissions for diabetic ketoacidosis in ethnic minority groups in a city hospital. Metabolism 56: $172-178$

3. Pinero-Pilona A, Raskin P (2001) Idiopathic Type 1 diabetes. J Diabetes Complications15: 328-335.

4. Kim MK, Lee SH, Kim JH, Lee JI, Kim JH, et al. (2009) Clinical characteristics of Korean patients with new-onset diabetes presenting with diabetic ketoacidosis. Diabetes Res Clin Pract 85: e8-11.

5. Umpierrez GE, Smiley D, Kitabchi AE (2006) Narrative review: ketosis-prone type 2 diabetes mellitus. Ann Intern Med144: 350-357.

6. Maldonado MR, Otiniano ME, Lee R, Rodriguez L, Balasubramanyam A (2004) Characteristics of ketosis-prone diabetes in a multiethnic indigent community. Ethn Dis 14: 243-249.

7. Balasubramanyam A, Zern JW, Hyman DJ, Pavlik V (1999) New profiles of diabetic ketoacidosis: type 1 vs type 2 diabetes and the effect of ethnicity. Arch Intern Med 159: 2317-2322.

8. Sobngwi E, Mauvais-Jarvis F, Vexiau P, Mbanya J-C, Gautier JF (2002) Diabetes in Africans. Part 2: Ketosis-prone atypical diabetes mellitus. Diabetes Matab 8: 5-12.

9. Yan SH, Sheu WH, Song YM, Tseng LN (2000) The occurrence of diabetic ketoacidosis in adults. Intern Med 39: 10-14

10. Sobngwi E, Gautier JF (2002) Adult-onset idiopathic Type I or ketosis-prone Type II diabetes: evidence to revisit diabetes classification. Diabetologia 45 283-285.

11. Pinero-Pilona A, Litonjua $P$, Aviles-Santa L, Raskin $P$ (2001) Idiopathic type 1 diabetes in Dallas, Texas: a 5-year experience. Diabetes Care 24: 1014-1018.

12. Bouguerra R, Alberti H, Salem LB, Rayana CB, Atti JE, et al. (2007) The globa diabetes pandemic: the Tunisian experience. Eur J Clin Nutr 61: 160-165.

13. Mauvais-Jarvis F, Sobngwi E, Porcher R, Riveline JP, Kevorkian JP, et al (2004) Ketosis-prone type 2 diabetes in patients of sub-Saharan African origin clinical pathophysiology and natural history of beta-cell dysfunction and insulin resistance. Diabetes 53: 645-653.

14. Tanaka K, Moriya T, Kanamori A, Yajima Y (1999) Analysis and a long-term follow up of ketosis-onset Japanese NIDDM patients. Diabetes Res Clin Pract 44: $137-146$

15. Banerji MA, Chaiken RL, Huey H, Tuomi T, Norin AJ, et al. (1994) GAD antibody negative NIDDM in adult black subjects with diabetic ketoacidosis and increased frequency of human leukocyte antigen DR3 and DR4. Flatbush diabetes. Diabetes 43: 741-745

16. Umpierrez GE, Kitabchi AE (2003) Diabetic ketoacidosis: risk factors and management strategies. Treat Endocrinol 2: 95-108.

17. Umpierrez GE, Smiley D, Robalino G, Peng L, Gosmanov AR, et al. (2010) Lack of lipotoxicity effect on \{beta\}-cell dysfunction in ketosis-prone type 2 diabetes Mellitus. Diabetes Care 33: 626-631.

18. Umpierrez GE, Smiley D, Gosmanov A, Thomason D (2007) Ketosis-prone type 2 diabetes: effect of hyperglycemia on beta-cell function and skeletal muscle insulin signaling. Endocr Pract 13: 283-290.

19. Matsui J, Tamasawa N, Tanabe J, Kasai N, Murakami H, et al. (2005) Clinica characteristics of Japanese youth-onset type 2 diabetes with ketonuria. Diabetes Res Clin Pract 70: 235-238.

20. Sobngwi E, Choukem SP, Agbalika F, Blondeau B, Fetita LS, et al. (2008) Ketosis-prone type 2 diabetes mellitus and human herpesvirus 8 infection in sub-saharan africans. JAMA 29: 2770-2776

21. Maldonado MR, Otiniano ME, Cheema F, Rodriguez L, Balasubramanyam A (2005) Factors associated with insulin discontinuation in subjects with ketosisprone diabetes but preserved cell function. Diabet Med 22: 1744-1750.

22. Hsin Yu E, Guo HR, Wu TJ (2001) Factors associated with discontinuing insulin therapy after diabetic ketoacidosis in adult diabetic patients. Diabet Med18: 895-899.

23. Umpierrez GE, Clark WS, Steen MT (1997) Sulfonylurea treatment prevents recurrence of hyperglycemia in obese African-American patients with a history of hyperglycemic crises. Diabetes Care 20: 479-483.

24. Li JK, Chan JC, Zimmet PZ, Rowly MJ, Macley IR, et al. (2000) Young Chinese adults with new onset of diabetic ketoacidosis - clinical course, autoimmune status and progression of pancreatic beta cell function. Diabet Med 17: 295 298.

25. Ramos-Román MA, Piñero-Piloña A, Adams-Huet B, Raskin PJ (2006) Comparison of type1, type 2 and atypical ketosis-prone diabetes at 4 years of diabetes duration. J Diabetes Complications 20: 137-144

26. Harzallah F, Ben Brahim A, Laadhar L, Feki M, Zitouni M, et al. (2010) Ketosisonset diabetes in Tunisian adults: immunological markers and beta-cell function. East Mediterr Health J 16: 70-74. 